\title{
Leseliste Top 5: Controlling und Datenqualität
}

In diesem Teil möchten wir Ihnen regelmäßig fünf besonders lesenswerte Bücher zu einem bestimmten Themengebiet vorstellen:

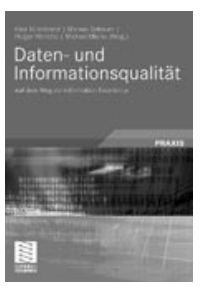

Hildebrand, K./ Mielke, M./ Gebauer, M./ Hinrichs, H. (Hrsg.):

Daten- und Informationsqualität Auf dem Weg zur Information Excellence

Vieweg+Teubner, Wiesbaden 2008, 415 Seiten, ISBN: 3834803219, Preis 39,90€

Die Herausgeber haben mit diesem Band ein umfassendes und aktuelles Werk zum Thema Daten- und Informationsqualität geschaffen. Der Herausgeberband ist gegliedert in Grundlagen der Informationsqualität, Methoden/Techniken/Tools und Organisation. Diese wichtigen Themenbereiche werden von hochkarätigen Autoren wissenschaftlich fundiert aufgearbeitet und in der praktischen Anwendung dargestellt.

Tipp: Die dargebotenen Praxisfälle machen das Buch für (IT-)Manager und Datenspezialisten (bspw. aus Banken und Versicherungen) interessant. Aufgrund der Aufarbeitung des aktuellen Stands der Forschung hat es darüber hinaus auch für die Lehre und Forschung Relevanz.

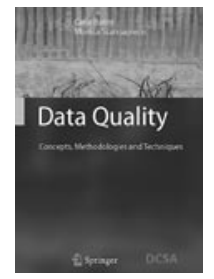

Batini, C./

Scannapieco, M.:

Data Quality

Concepts, Methodologies and Techniques

Springer, Berlin 2006, 262 Seiten, ISBN: 3540331727, Preis 53,45€ (Englisch)

Dieses Buches gibt eine systematische Einführung in die wichtigsten Facetten des Themas Datenqualität. Zunächst werden die verschiedenen Dimensionen von Datenqualität und deren Bedeutung in unterschiedlichen Bereichen dargestellt. Im Anschluss wird der Status quo des Datenqualitätsmanagements durch umfangreiche Ausführungen zu Techniken und
Aktivitäten (z. B. Datenqualitätsforschung, Data Mining, Machine Learning) aufgearbeitet. Das Buch schließt mit einer Beschreibung und kritischen Betrachtung praktischer Methoden zur Messung und Verbesserung von Datenqualität.

Tipp: Durch die Kombination theoretischer Ansätze und praktischer Lösungen eignet sich dieses Buch für Lehre und Praxis gleichermaßen.

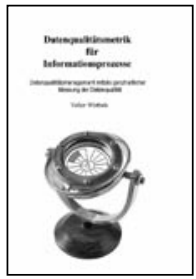

Würthele, V.:

Datenqualitätsmetrik für Informationsprozesse

Datenqualitätsmanagement mittels ganzheitlicher Messung der Datenqualität

Books on Demand GmbH, 2003, 176 Seiten, ISBN: 3833403454 , Preis $32,00 €$

Der Autor stellt in seinem Buch ein ganzheitliches Metriksystem zur Messung und Kontrolle von Datenqualität vor. Dieses berücksichtigt auch schlecht quantifizierbare weiche Faktoren, die Zeitabhängigkeit der Qualität sowie Effekte der Modellbildung.

Tipp: In dem Buch finden sich Download-Hinweise auf freiverfügbare Simulationsprogramme. Diese ergänzen das Metriksystem, indem sie das Auffinden derjenigen Workflowkomponenten oder Schnittstellen erleichtern, deren Verbesserung den größten Einfluss auf die Datenqualität insgesamt hat.

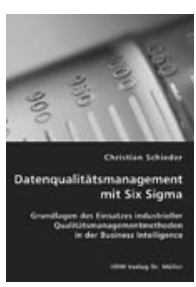

Schieder, C.:

Datenqualitätsmanagement mit Six Sigma

Grundlagen des Einsatzes industrieller Qualitätsmanagementmethoden in der Business Intelligence

VDM Verlag Dr. Müller, Saarbrücken 2007, 156 Seiten, ISBN: 3836426587, Preis 59,00€

Der Autor dieses Buches erschließt die bereits im Qualitätsmanagement der Konsumgüterindustrie etablierte Six SigmaMethodik für das Datenqualitätsmanage- ment. Mit Fokus auf Business Intelligence gibt dieses Buch einen Überblick über die aktuellen Ansätze im Datenqualitätsmanagement, führt den Leser in den Six Sigma-Ansatz ein und stellt die Anpassung des Ansatzes an das Datenqualitätsmanagement vor.

Tipp: Das Buch richtet sich an Entscheider und Projektverantwortliche in der IT, $\mathrm{ClOs}$, Informationsmanager und alle interessierte Praktiker, die Datenqualitätsprobleme lösen wollen.

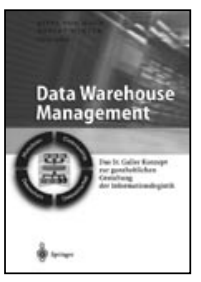

Winter, R。/ von Maur, E. (Hrsg.):

Data Warehouse Management

Das St. Galler Konzept zur ganzheitlichen Gestaltung der Informationslogistik

Springer, Berlin 2008, 359 Seiten, ISBN: 3540005854, Preis 54,95€

Dieser Sammelband stellt die Themenbereiche Datenqualitäts- und Metadatenmanagement, Datenschutz und Datensicherheit als Herausforderungen des Data Warehouse Managements heraus. Die Autoren gehen dabei auch auf die mit dem Customer Relationship Management, der Enterprise Application Integration und dem Knowledge Management verbundenen Synergiepotenziale ein.

Tipp: Die Autoren zeigen fundierte Konzepte und praktikable Lösungen auf, die im zweiten Teil des Buches anhand von Umsetzungen in der Praxis verdeutlicht werden.

Natalie Kink, München 